\title{
Poster Presentation
}

\section{MS11.P01}

\author{
Powder diffraction and DFT studies of synthetic insect pheromones
}

$\underline{\text { A. Fitch }}{ }^{1}$, L. Brooks ${ }^{1,2}$, P. Pattison ${ }^{3,4}$, M. Brunelli, ${ }^{1,5}$, G. Jones $^{2}$

${ }^{1}$ ESRF, Grenoble, France, ${ }^{2}$ Department of Chemistry, Keele University, Staffordshire, UK, ${ }^{3}$ Crystallography Competence Centre, EPFL, Lausanne, Switzerland, ${ }^{4}$ Swiss-Norwegian Beamlines, ESRF, Grenoble, France, ${ }^{5}$ Institut Laue Langevin, Grenoble, France

Eight chiral monomethyl alkanes have been synthesized and their crystal structures solved from high resolution powder diffraction patterns collected using synchrotron radiation. Such compounds have been seen to act as recognition pheromones in social insects. Carbon chain lengths varied from $C_{25}$ to $C_{31}$ with the methyl group at various positions along the chain. One compound, (S)-7methylnonacosane, also formed a low quality single crystal from which its structure was solved and refined. Although the powder diffraction patterns were good enough to solve the crystal structures, using the standard technique of real-space simulated annealing, with near-linear molecules, chemically unreasonable distortions occurred during Rietveld refinement unless many distance, angle and torsion restraints were imposed. From the results of the single-crystal structure it is evident that there is a gentle bend in the molecule close to the position of the methyl side chain. Thus imposing a near-linear molecule is incorrect, and giving sufficient freedom in the Rietveld refinement to allow the molecule to bend leads to an inaccurate structure. In short, the correct structure must be known to judge the strength of the restraints to apply, and this must vary at different parts of the chain. As an alternative to refining the structure from the powder diffraction data, the structures have been optimized via dispersion-corrected DFT calculations (Van der Streek and Neumann, 2010) starting from the powder structures. Agreement between the single-crystal and DFT-minimized structures is satisfactory, with an rms distance of $0.054 \AA$ and a maximum distance of $0.132 \AA$ when comparing carbon atoms positions. With this approach, a well defined structure optimization strategy is used for all the crystal structures. The molecules studied fall into two packing types, (both with monoclinic symmetry), depending on the position of the methyl group on the main carbon chain relative to the overall chain length.

[1] J. van de Streek, M. Neumann, Acta Cryst., (2010), B66, 544-558

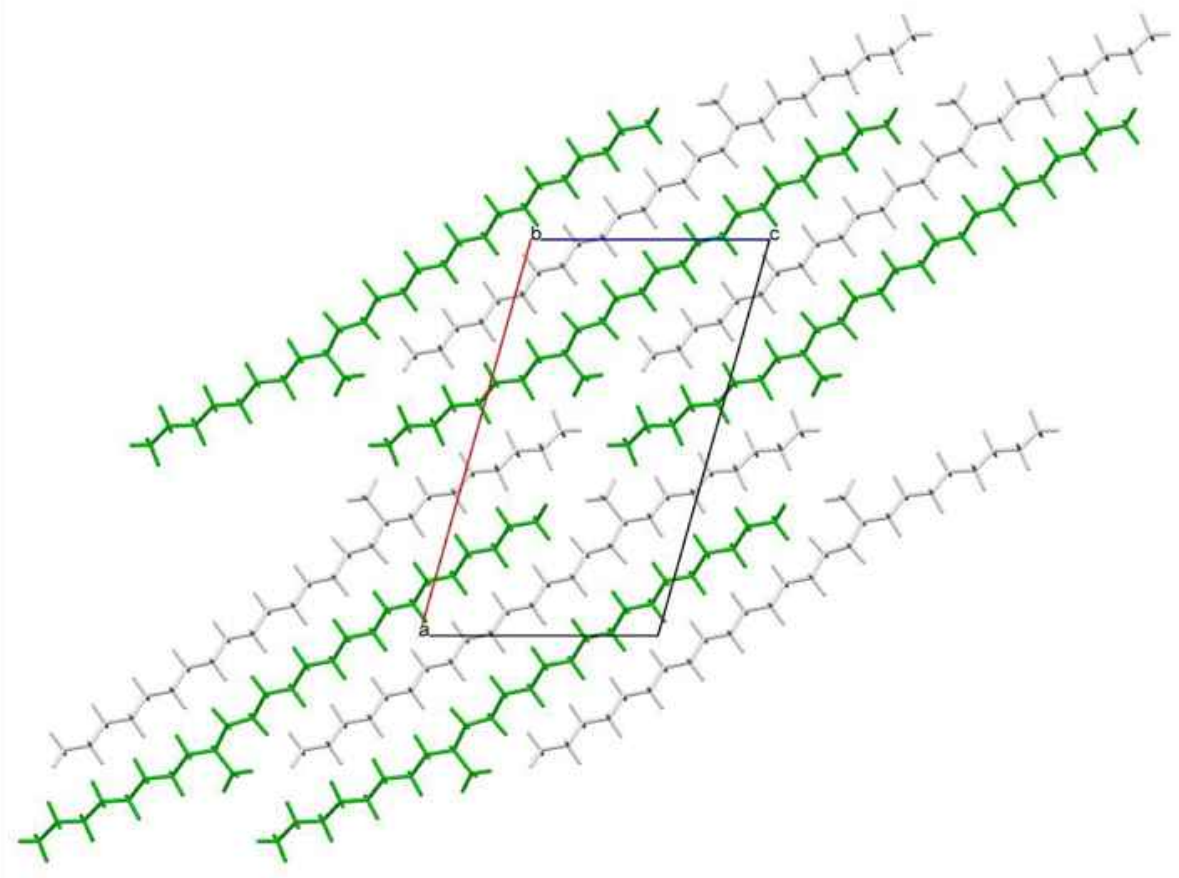

Keywords: Synchrotron powder diffraction, DFT optimization 